\title{
How Clause-bounded is the Scope of Universals?
}

\author{
Donka F. Farkas \\ University of California, Santa Cruz
}

\author{
Anastasia Giannakidou \\ University of Groningen
}

\section{The problem}

This paper addresses a particular problem in the constellation of issues we name "scope limits on quantifiers and variables". The problem concerns the limits on the inverse scope of a distributive universal DP over an indefinite. We assume here a semantic notion of scope according to which an expression $a$ is in the scope of an expression $b$ iff the interpretation of $a$ is affected by the semantic contribution of $b$. (For discussion, see Farkas (1995).) For the cases we are interested in here the relation is defined in (1).

(1) A variable $y$ contributed by an indefinite is within the scope of a distributive universal binding a variable $x$ iff the interpretation of $y$ co-varies with the interpretation of $x$.

The relation between scope and syntactic hierarchy is of particular interest to both semanticists and syntacticians. The issue concerns the relation between surface c-command (defined in terms of branching nodes) and semantic scope. An expression $a$ is said to have direct scope over an expression $b$ iff $b$ is in the scope of $a$ and $a$ c-commands $b$ in Surface Structure. An expression $a$ is said to have inverse scope over an expression $b$ iff $a$ has scope over $b$ and $b$ c-commands $a$ in Surface Structure.

Turning now to scope limits, we take it as established that the direct scope of universals is unlimited:

(2) A universal may take scope over any indefinite in its c-commanding domain.

Concerning inverse scope, it is well known that clause-internal inverse scope of a universal over an indefinite is possible. Thus, example (3a) is scopally ambiguous, the reading in $c$. involving the indefinite co-varying with the universal.

(3) a. A member of the Expose the Right group attended every public event held in Iowa or New Hampshire. (The New Republic, Feb. $19,1996)$

b. a member of the group > every public event

c. every public event > a member of the group

It is also well known that the inverse scope of universals is not unlimited. 
Thus, (4), unlike (3), has only the (absurd) reading in (4b); the (sensible) reading in $(4 c)$ is lacking:

(4) a. A politician said that every city should be the capital.

A politician said that the President lives in every city.

b. a politician > every city

c. $\quad *$ every city $>$ a politician

Because of the contrast between (3) and (4), it is standardly assumed in the literature that (5) holds. (see Farkas (1981), Fodor and Sag (1982), Abush (1994), Beghelli (1993), Szabolcsi (1995).)

(5) An element in a clause $S$ cannot be within the scope of a universal quantifier in a clause $S^{\prime}$ if $S$ c-commands $S^{\prime}$.

Universals contrast in this respect with indefinites, whose inverse scope is unbounded. Yet, there are counterexamples to (5), such as (6a), which has both the direct scope reading in (6b) and the inverse scope reading in (6c):

a. A student made sure that every invited speaker had a ride.

b. a student $>$ every speaker c. every speaker $>$ a student

Under the c. reading, invited speakers are assigned students who take care of their needs. The universal in this case is said to have extrawide scope. ${ }^{1}$ Extrawide scope then involves inter-clausal inverse scope of a universal over an indefinite. The extrawide scope reading (6c) is robust enough to warrant attention.

In this paper we will investigate, based on data from Greek, the conditions that allow a universal to take extrawide scope over an indefinite. We will then propose an account based on an extension of the notion of co-argument. The account is implemented in the indexical semantics framework of Farkas (1995). We conclude with a review of open problems and a discussion of the implications of our findings for the treatment of scope phenomena in general.

\section{Extrawide scope is not always an illusion}

We first establish that extrawide scope is real. This is an issue since Fox and Sauerland (1995) argue the contrary. They discuss examples involving apparent unexpected wide scope readings of universals over indefinites in generic contexts such as (7).

(7) a. In general, a guide ensures that every tour to the Louvre is fun.

b. In general, I give a tourist every leaflet. 
Here we are interested in (7a), which has a reading under which the indefinite co-varies with the universal and therefore the universal appears to take extrawide scope.

Fox and Sauerland argue that the apparent wide scope of the universal over the indefinite in $(7 \mathrm{a})$ is due to the fact that the indefinite is bound by the generic operator, combined with the assumption that the situations quantified over by the operator may involve single guide/tour (tourist/leaflet) pairs. They conclude that cases when universals appear to take unexpected wide scope over indefinites always involve the indefinite being bound by an implicit or explicit adverb of quantification, and therefore that the generalization in (5) can be maintained.

While we accept Fox and Sauerland's analysis of $(7 \mathrm{a}, \mathrm{b})$, we argue that matters cannot be laid to rest because cases of extrawide scope arise not only in generic but also in episodic contexts, and in such contexts the effect cannot be attributed to a matrix operator binding the indefinite. Thus, contrary to what Fox and Sauerland (1995) predict, the episodic version of their (7a), given in (8), is also judged as scopally ambiguous:

(8) Yesterday, a guide made sure that every tour to the Louvre was fun.

Note also that in the c. reading of (6a) above, the universal takes extrawide scope with respect to the indefinite (and as a result students co-vary with invited speakers), and yet the sentence is not generic. In Greek, the extrawide scope reading of the counterpart of (6a), given in (9), is even more robust.

Kapjos fititis frondise kathe proskeklimenos omilitis na exi metafora. some student took-care.PERF every invited speaker subj has transportation 'Some student made sure that every invited speaker got a ride.'

The perfective aspect of the matrix verb in (9) is a morphological signal of the episodic nature of this sentence.

Note now that in Greek, just like in English, the universal cannot have inverse scope out of the complement of the verb leo 'say', and therefore (10), just like (4), is unambiguous despite the pragmatic oddity of the narrow scope reading of the universal.

(10) a. Kapjos politikos ipe oti kathe poli prepi na jini protevusa. some politician said that-ind. every city should subj be capital 'Some politician said that every city should be the capital.'

b. some politician > every city

c. $\quad{ }^{*}$ every city $>$ some politician

Based on the ambiguous status of (6a), (8) and (9) we conclude that a distributive universal may have extrawide scope over an indefinite in episodic sentences and therefore that extrawide scope is not always an illusion. Based on the contrast between (6) and (9) on the one hand, and (5), (10) on the 
other, we conclude that the phenomenon of extrawide scope out of an embedded clause is restricted. The next task is to determine what the nature of this restriction is. In order to do that, we turn to a more detailed examination of the cases when extrawide scope is possible and of those when it is not.

\section{The role of the semantics of the matrix predicate}

We show below that one of the crucial factors involved is the semantic nature of the matrix predicate. In what follows we restrict our attention to Greek. Under A we list cases when extrawide scope is possible, and under B, cases when it is not.

\section{A. Cases when extra wide scope is possible}

- $\quad$ Subjunctive complements of the verbs in (11a):

(11) a. frontizo 'take care', 'thelo' want, 'kanonizo' make sure

b. Kapjos kathijitis kanonise kathe fititis s' afti ti lista na vri dhoulja

some professor arranged every student in this the list subj find job 'Some professor arranged every student on this list finds a job.'

c. $\quad$ some prof. $>$ every student; d. every student $>$ some prof.

- $\quad$ Purpose clauses (subjunctive):

(12) a. rithmizo 'arrange', taktopio 'fix', diasfalizo 'ensure'

b. Kapjos kathijitis rithmise etsi ta pragmata oste kathe taksi na some professor arranged so the things that even class subj ine proetimasmeni ja tis eksetasis.

be prepared for the exam

'Some professor arranged things so that every class be prepared for the exam.'

c. some prof. $>$ every class; d. every class $>$ some prof.

B. Cases when extra wide scope is not possible

- Subjunctive complements of the verbs in (13a):

(13) a. efxome 'wish', protino 'suggest', parakalo 'ask for'

b. Kapjos politikos protine kathe poli na jini protevusa some politician suggested eveny city subj become capital 'Some politician suggested every city to become the capital.'

c. some pol. > every city; d. $\quad$ d. every city $>$ some pol.

- Indicative complements of the verbs in (14)

(14) a. leo 'say', isxirizome 'claim', onirevome 'dream'

b. Kapjos fititis onireftike oti kathe kathijitis tis sxolis apolithike some student dreamt that every professor the department got-fired 'Some student dreamt that every professor in the department got 
fired.'

c. $\quad$ some student $>$ every prof.; d. ${ }^{*}$ every prof. $>$ some student

Note that mood cannot be the distinguishing factor between the cases in A and the cases in B: although extrawide scope is possible only out of embedded subjunctive clauses, there are subjunctive clauses which block it, namely those in (13).

We argue below that the relevant difference involves details of the lexical semantics of the matrix predicate. Specifically, it concerns the fact that for the cases under $\mathrm{A}$, the matrix predicate assigns to its $\mathrm{Su}$ argument the role of initiator of the eventuality denoted by the clause that contains the critical universal, while for the cases under B this is not so. The initiator role is defined in (15) (see Farkas 1988)):

An individual $i$ is the initiator of an eventuality $e$ iff $i$ brings $e$ about (in some world or worlds). In this case $i$ is said to be in the RESP relation with $e$.

The semantic role of the initiator and the RESP relation were shown to play a role in the semantics of obligatory control and obviation (cf. Farkas (1988) and Farkas (1992)). We show below that these notions are relevant to characterizing extrawide scope as well.

First, some brief comments are in order as regards the relation between initiators and Agents. If $i$ is the initiator of $e$ and also a participant in $e$, then $i$ is one of the arguments of the predicate that names $e$, namely the Agent. The initiator of an event $e$, however, need not be a participant in $e$, and consequently, it need not figure in the argument structure of the predicate naming $e$. In such cases $i$ is the external Agent of $e$, defined as in (16).

(16) If $i$ is the initiator of $e$ but it is not a participant in $e, i$ is the external Agent of $e$.

To exemplify, consider the sentences below ((17b) is from Farkas (1988)):

(17) a. John put the sale sign in the shopwindow in order to attract customers.

b. The store had a sale sign in the shopwindow in order to attract customers.

In (17a), John, the subject of the matrix clause, is the initiator of the eventuality denoted by its clause, as well as its Agent; he is also the controller of the understood subject of the purpose clause. In (17b) on the other hand, the understood subject of the purpose clause is interpreted as having brought about the eventuality denoted by the matrix but in this case without being a direct participant in this eventuality. The understood Su of the purpose clause in (17b) is the external Agent of the eventuality denoted by the matrix.

Going now back to our cases under $\mathrm{A}$ and $\mathrm{B}$, note that in the A-cases, 
when extrawide scope is possible, the matrix indefinite is the external Agent of the eventuality denoted by the clause containing the critical universal. In the examples under $\mathrm{B}$, on the other hand, this is not the case. Thus, when you arrange, fix or make sure that an eventuality obtains, you have to perform an action meant to bring that eventuality about. When you wish, or suggest that something happen or when you say or dream that something happens, no such action is entailed. In the cases under $\mathrm{A}$, the referent of the matrix indefinite is entailed to have taken action (or contemplate taking action) in order to bring about the embedded eventuality, while in the cases under B no such entailment is present. The descriptive generalization that characterizes the extrawide scope cases we looked at so far is stated in (18):

First generalization

A distributive universal $\mathrm{DP}_{\mathrm{i}}$ may have extrawide scope over an indefinite $\mathrm{DP}_{\mathrm{j}}$ when the referent of $\mathrm{DP}_{\mathrm{j}}$ is the external Agent of the eventuality denoted by the clause containing $\mathrm{DP}_{\mathrm{i}}$.

This generalization correctly predicts the contrast in (19) and (20), given that in (19a) the matrix Su can be understood as the initiator of the eventuality denoted by the complement, while in (20a) it cannot:

(19) a. Kapjos mixanikos kanonise kathe ethusa s' afto to ktirio na exi some engineer arranged every room in this the building subj has kali akustiki good acoustics

'Some engineer arranged that every room in this building have good acoustics.'

(20) a. Kapjos kathijitis ithele kathe ethusa s' afto to ktirio na exi some professor wanted every room in this the building subj has kali akustiki

good acoustics

'Some professor wanted every room in this building to have good acoustics.'

b. $\quad$ some professor $>$ every room; * every room $>$ some professor

In (19a), the referent of the indefinite is naturally understood as bringing about the eventuality in the complement, hence it is the external Agent of that eventuality. In (20a), on the other hand, given standard assumptions about professors not having much direct influence on acoustics of classrooms, the indefinite is not interpreted as being the external Agent of the eventuality denoted by the complement. The possibility of extrawide scope parallels this difference. 


\section{Extended co-arguments}

Let us restate the problem as it appears now. First, clause internal inverse scope of a distributive universal over an indefinite is possible (cf. (3)). Second, inter- clausal inverse scope, i.e., extrawide scope, is allowed when the matrix indefinite is the external Agent of the embedded eventuality. The following two questions arise at this point: (i) why is the restriction against inter-clausal inverse scope relaxed under this particular condition?, and (ii) more generally, is the restriction against inverse scope of universals sensitive to the syntactic relation of clause-mateness or to the semantic relation of co-argumenthood? ${ }^{2}$

We start answering these questions by looking at the initiator role and the RESP relation in further detail. As discussed in work on control and obviation, when $i$ is the external Agent of an eventuality $e, i$ may bring $e$ about by acting directly on a participant in $e$. We call this participant the affected argument. The affected argument is canonically realized as the subject of the embedded clause. ${ }^{3}$ Two points are crucial for us. First, that the external Agent of $e$ acts on the affected argument when bringing $e$ about, and second, that the affected argument is canonically realized as the subject of the embedded clause denoting $e$.

Going back to extrawide scope now, note that the cases allowing it are characterized by the following two properties: (i) as stated in (18), the indefinite is the external Agent of the eventuality denoted by the embedded clause; (ii) the universal is the affected argument. The new generalization is given in (21):

\section{Second generalization}

A distributive universal $\mathrm{DP}_{\mathrm{i}}$ that realizes a participant in an eventuality $e$ may have extrawide scope over an indefinite $\mathrm{DP}_{\mathrm{j}}$ when the referent of $\mathrm{DP}_{\mathrm{i}}$ is the external Agent of $e$, and the argument realized by $\mathrm{DP}_{\mathrm{i}}$ is the affected argument.

The crucial observation here is that when a professor arranges that a student gets a job, the student is affected by the actions of the professor, and if several students are involved the professor may need to take several specific actions, one per student.

Our claim is that the extrawide scope effect is due to the fact that, given the semantics of the sentence, the referent of the indefinite may be understood as acting upon the argument denoted by the universal. More generally put, extrawide scope is possible because there is a direct semantic relation between the two arguments involved in virtue of which they act as if they were co-arguments. The crucial semantic characteristic of co-arguments is that they are both relata in the relation named by the predicate. In the case of an external Agent and the affected argument, they share with co-arguments the property of being relata in a semantic relation but in this case the existence of the relation is mediated by the semantics of the predicates the two expressions are arguments of. The external Agent and the affected 
argument are extended co-arguments in the sense defined in (22):

(22) Extended Co-anguments

In a configuration like (i),

(i) $(\mathrm{P}(\mathrm{x},[\mathrm{Q}(\mathrm{y}, \ldots], \ldots)$

$x$ and $y$ are extended co-arguments iff, given the semantics of $\mathrm{P}$ and $\mathrm{Q}$, there is an eventuality $e^{\prime}$ in which the referents of $x$ and $y$ are co-participants. In this case there must be a direct relation $\mathbf{R}$ that obtains between $x$ and $y$.

Clause-bounded inverse scope is possible because the two expressions involved in the scope relation are co-arguments. Extrawide inverse scope is possible when the two expressions involved are extended co-arguments. In the cases we looked at so far, these involved external Agents and affected arguments.

The common feature of clause-bounded and extrawide scope is that the two scopally related expressions are co-participants in some eventuality, hence also relata in a semantic relation $\mathrm{R}$. In the face of this, we arrive at the final generalization in (23):

\section{Final generalization}

Extrawide scope of a distributive universal over an indefinite is possible iff the two arguments are co-participants in some eventuality $e$ in virtue of the lexical semantics of the predicates involved. In this case the two arguments are relata in some relation $R$.

The moral to be drawn from the above discussion is that what makes inverse scope possible is not syntactic clause-mateness but semantic co-argumenthood.

\section{Further predictions}

\subsection{Non-subject universals cannot have extrawide scope}

Given (23) and the fact that affected arguments are canonically realized as embedded subjects, we predict that non-subject universals cannot have extrawide scope even if the matrix predicate is of the right type. The data in (24) show that this prediction is borne out. These sentences cannot be interpreted with the indefinite within the scope of the universal:

(24) a. Kapjos kathijitis kanonise o proedros na parakoluthisi kathe ekdilosi

some professor arranged the president subj attends every event 'Some professor arranged that the president attend every event.'

b. Kapjos kathijitis frondise i gramateas na dosi tis etisis se kathe fititi

some professor took-care the secretary subj gives the applications 
to every student

'Some professor made sure that the secretary gives the applications to every student.'

c. $\quad$ some prof. $>$ every event ${ }^{*}$ every event $>$ some prof.

d. $\quad$ some professor $>$ every appl. * every appl. $>$ some professor

So far then we accounted for the possibility of inverse scope in the case of arguments, and for the possibility of extrawide scope when the indefinite is an external Agent and the distributive universal is an affected argument. The two cases share the property that the indefinite is an argument of a relation $\mathbf{R}$ that is distributed over the domain of the universal.

\subsection{Perception verbs}

The core claim behind (23) is that inverse scope is possible only in case the two expressions involved are semantic co-arguments. The semantic coargumenthood condition is trivially satisfied in mono-clausal cases, when the two expressions are syntactic co-arguments as well. The condition on co-argumenthood is less trivially satisfied inter-clausally just in case the indefinite and the distributive universal are extended co-arguments. In that case, syntactic and semantic co-argumenthood diverge. We saw one such case in some detail, involving external Agents and affected arguments. Our account does not rest on these particular roles, and therefore we expect other cases of mismatch between semantic and syntactic co-argumenthood to behave in a similar manner.

We examine briefly another class of cases, namely perception verbs. In Greek, just like in English, such verbs license ECM constructions, where the embedded subject is marked Accusative:

(25) a. Kapja nosokoma idhe kathe astheni na perni to farmako tu some nurse saw every patient.acc subj take the medicine his 'Some nurse saw every patient take his medication.'

b. $\quad$ some nurse $>$ every patient; c. every patient $>$ some nurse

In such sentences, in both English and Greek, a distributive universal embedded subject may have extrawide scope over an indefinite matrix subject, hence $(25 \mathrm{a})$ has the readings in $(25 \mathrm{~b}, \mathrm{c})$. Our account predicts this ambiguity on semantic grounds since, as has long been noted about perception verbs, the relation denoted by the matrix predicate must hold between the matrix subject and the accusative subject. Because of this requirement, the two subjects will be extended co- arguments in our sense, and (23) predicts the possibility of inverse scope. We also predict that non-subject universals in the embedded clause cannot have extrawide scope. The non-ambiguous status of (26a) shows that this prediction is correct.

(26) a. Kapjos kathijitis akuse ton Pavlo na perni sinendefksi kathe fititi 
some professor heard the Paul.acc subj take interview every student 'Some professor heard Paul interview every student.'

b. some $>$ every c. ${ }^{*}$ every $>$ some

Greek does not license ECM with consider-type verbs. Yet, just like in English, consider may be construed with a small clause in which case extrawide scope is possible, given that in that configuration, subject and direct object are syntactic and semantic co-arguments:
a. Kapjos kathijitis theori kathe fititi idhiofiia some professor considers every student genius 'Some professor considers every student a genius.'
b. some > every
c. every > some

Hornstein (1995) makes the observation that in English small clause-consider contrasts with ECM-consider in that the former but not the latter allows the accusative universal to scope over the matrix subject. This is to be expected in view of the fact that the semantics of ECM-consider is close to the semantics of think and therefore the two subjects are not extended coarguments.

\section{Implementation in an indexical theory of scope}

The facts presented above are not naturally amenable to a purely syntactic account of scope. In its pristine form, such an account rests on the following two assumptions: (i) DPs c-command their scope at LF. Consequently, if an indefinite is interpreted as co-varying with a universal, the latter must c-command the former at LF. (ii) DPs reach their LF positions via QR, a syntactic movement rule which adjoins DPs to a higher clausal node. Under these assumptions, the instance of $\mathrm{QR}$ responsible for raising quantificational DPs will have to be clause-bounded since normally the scope of such DPs is clause -bounded. Clauses would therefore have to act as barriers to this movement. When the DP is a distributive universal, however, QR would have to be allowed to cross one clause boundary in case the conditions in (23) are met, namely when the universal DP and one of the arguments in the matrix are extended $c_{0}$-arguments. ${ }^{4}$ In just these cases then, the clause boundary should not count as a barrier. Given that there is no independently justified syntactic characterization of the clauses in question, the syntactic nature of the phenomenon is in serious doubt. Recall that an appeal to mood will not do, since there are subjunctive clauses that act as barriers for extrawide scope, while others do not. An appeal to time dependency relations between matrix and complement would not help either, since the complement of protino 'suggest' does not allow extrawide scope of its universal subject, while that of kanonizo 'make sure' does, and yet, both complements have future time reference with respect to the matrix. Note also that subjunctive clauses 
allowing extrawide scope do not differ from those that do not with respect to the status of their subjects either. Finally, simply claiming that the clause boundary of certain complements ceases to act as a barrier to QR would fail to predict the restriction to subjects discussed in 5.1. We conclude that a purely structural account is not feasible.

Beghelli and Stowell (1995) propose a semantically sophisticated version of $Q R$, which raises a DP to a particular LF position that is compatible with the semantic feature of the DP in question. Though sensitivity to semantic factors is built into this system, the data discussed here are still problematic: whether a universal distributive DP moves to the head of the appropriate semantic projection within its own clause or whether it may go one clause up would have to depend on semantic and lexical details that do not normally interfere with the operation of syntactic rules.

We turn now to an account of these facts within the indexical approach to scope proposed in Farkas (1995), where both assumptions (i) and (ii) above are given up. Under this approach, syntactic configuration does not determine scope relations univocally. The crucial relevant aspects of the theory are listed below under (a)-(f).

(a) Semantic interpretation operates on semantic structures. It is at this level that scopal relations are determined.

(b) Indefinite DPs contribute to the semantic structure a variable and a restrictive expression contributed by the Descriptive Content of the DP. ${ }^{5}$ Following Heim (1982) and Kamp (1981), we assume that the existential force of indefinites is a consequence of the definition of truth of expressions that may contain free variables:

(28) Let $a$ be an expression that may contain free variables. $a$ is true iff there is an assignment function $f$ that satisfies the conditions of $a$.

(c) Quantification at the semantic structure level involves a Restrictor, a Quantifier and a NS, as in (29):

\section{Restrictor Q NS}

The Restrictor identifies a set of cases; the whole expression is true iff Q-many of these cases are such that the NS is true of them. The cases identified by the Restrictor constitute the logical subject, while the expression in the NS is the logical predicate. The predication relation between the Restrictor and the NS may be distributive or collective.

(d) A DP whose Determiner is a distributive universal (that is, every or each in English, kathe and o kathenas in Greek respectively) contributes a variable $x$ and a restrictive expression $e_{R}$ contributed by the Descriptive Content (DC) of the noun phrase. The semantic contribution of such a DP must form the Restrictor of a universal quantificational structure whose NS is predicated distributively over the set of cases introduced by the Restrictor. Because of the distributivity requirement of every and each we will call them 
strong distributives. ${ }^{6}$ The semantic structure of an expression containing a strong distributive is as in (30), where $e_{R}$ is the contribution of the DC of the universal DP, and $e_{N S}$ is the expression in the Nuclear Scope.

$$
\begin{array}{lll}
\text { Restrictor: } & \forall & \begin{array}{l}
\text { Nuclear Scope: } \\
x_{i}: e_{R}
\end{array}
\end{array}
$$

The role of the Restrictor here is to introduce a set of assignment functions $F$, one for each element of the witness set denoted by the universal DP. Because of the distributive nature of the predication, each such function must be extendable so as to satisfy the NS. Truth conditions of expressions of the form in (30) are given in (31):

(31) $a$ of the form in (30) is true iff there is an assignment function $f$ with the following property: for every $f^{\prime}$ that extends $f$ with respect to $x$ such that $f^{\prime}$ satisfies $e_{R}$, there is an assignment function $f^{\prime \prime}$ that extends $f^{\prime}$ such that $f^{\prime \prime}$ satisfies $e_{N S}$.

The notion of extension is defined in (32):

An assignment function $f^{\prime}$ extends an assignment function $f$ with respect to a (possibly empty) set of variables $X$ iff $f^{\prime}$ agrees with $f$ on all assignments except for the variables in $X$.

The notation $f$ ' $(f / x)$ will be used for ' $f$ ' extends $f$ with respect to $x$ '.

(e) A strong distributive DP introduces the set of evaluation functions $F^{\prime}$ that evaluate the Restrictor, as well as the set of functions $F^{\prime \prime}$ that extend the elements of $F^{\prime}$, and that evaluate the NS.

(f) A variable $y$ introduced by an indefinite is within the scope of a distributive universal introducing a variable $x$ iff the values of $y$ co-vary with those of $x$. This means that $y$ is evaluated by the functions in $F^{\prime \prime}$, which extend those in $F^{\prime}$ at least with respect to $y$. In this case $y$ is dependent on $x$. Dependent variables can be seen as Skolem functions whose range is given by the values of the variable they depend on.

Under these assumptions, the scope of $y$ with respect to $x$ is a matter of whether the value of $y$ is fixed independently of $x$, by $f$, or whether $y$ is dependent on $x$, in which case $y$ is evaluated by the functions in $F^{\prime \prime}$. This means that the functions in $F^{\prime \prime}$ extend those in $F^{\prime}$ with respect to $y$. The former option results in the wide scope reading of the indefinite with respect to the universal, and the latter in the narrow scope reading. Accessibility conditions define which assignment functions may evaluate a particular indefinite. Scope ambiguities are the result of several functions being accessible to a given indefinite. Ordinary indefinites may always have wide scope with respect to a universal because the initial function $f$ is always accessible to them.

Since structural conditions do not necessarily determine a unique 
to essentially semantic notions. Second, the scope limits of universals are genuinely different from that of indefinites, whose scope is unbounded. An adequate theory of scope has to account for this difference. Third, the contrast between the scope limits on distributive universals and non-distributive quantifiers on the one hand, and that between indefinites and quantificational DPs on the other supports the claim, made most recently in Szabolcsi (1995), that different DPs scope differently, depending on their semantic characteristics (see also Liu (1991), Ben-Shalom (1993).) Finally, the sensitivity of inverse scope to semantic factors of the type discussed above casts serious doubt on the possibility of a purely configurational treatment of scope.

\section{Endnotes}

* We would like to express our gratitude to our prompt and generous informants Artemis Alexiadou, Elena Anagnostopoulou and Cleo Condoravdi. Many thanks to Jason Merchant for judgements about English and helpful discussions, and to the audience of SALT VI for comments and suggestions, especially to Pauline Jacobson and Ken Safir. The second author's research was made possible by a visit to UCSC which was financed by the Dutch Organization for Scientific Research (NWO), grant R 43-13, which is hereby gratefully acknowledged.

1. For a brief discussion of extrawide scope, see Farkas (1995), from where (6) is taken.

2. This question, left open in Farkas (1981), echoes one raised by Bach and Partee (1980) concerning anaphora, and dealt with in Reinhart and Reuland (1993).

3. Accounting for the connection between affected arguments and subjects involves the interaction of Proto-Agent properties (as discussed in Dowty (1991)) and discourse prominence considerations, which we do not understand clearly enough at present and therefore leave outside the scope of this paper. The connection was invoked in Farkas (1992) to account for the subject orientation of obviation in Romance.

4. Problems raised for QR by the unbounded nature of the scope of indefinites, and the clause-bounded nature of the scope of distributives are discussed in detail in Farkas (1995).

5. We are assuming a logical syntax that uses restricted variables. For a discussion of the advantages of such a system for natural language semantics, see McCawley (1980)). Below, expressions whose role is to restrict the possible values of a variable will be prefixed by :.

6. The strong distributivity of these determiners is responsible for their inability to occur as subjects of collective predicates:

(i) * Every/each student gathered in the assembly room.

Each is more distributive than every in the sense that it requires a co-variant, i.e., an event or individual variable distributed over its domain. (see Vendler 


\section{Conclusions and open problems}

We start by reviewing some of the problems left open. The most salient one is the fact that only strong distributives may have extrawide scope. The scope of other quantifiers is strictly clause bounded: if one replaces every (Greek kathe) by all or many (Greek oli or poli respectively) in the examples that allow extrawide scope readings these readings disappear. We suggest that the ability of strong distributives to take extrawide scope should be connected to the ease with which they take clause-internal inverse scope. A comprehensive theory of scope has to account for the fact that inverse scope is a marked option in general, and that strong distributives avail themselves of it with more ease than other DPs. ${ }^{9}$ An account along the lines of Section 6 would limit inverse scope to cases where the functions introduced by the distributive are particularly salient, and would have to connect salience to strong distributivity.

The next problem involves intervention effects. It has been noted in the literature (most recently in Farkas and É. Kiss (1996)), that certain adverbs block inverse scope. As shown by the contrast between (37) and (38), an adverb like para poli 'very much' has the same effect in extrawide scope cases:

(37) a. Kapjos kathijitis ithele kathe ipopsifios na episkefti to panepistimio some professor wanted every candidate subj visit the campus

'Some professor wanted every candidate to visit the campus.'

b. some > every c. every > some

a. Kapjos kathijitis ithele para poli kathe ipopsifios na episkefti to some professor wanted very much every candidate subj visit the panepistimio

campus

'Some professor wanted very much every candidate to visit the campus.'

b. some $>$ every c. ${ }^{*}$ every $>$ some

At present we can only connect this problem to other unsolved mysteries. Thus, Giannakidou \& Quer (1995) and Giannakidou (in progress) note that the same adverbs block Neg Raising and the long distance licensing of NPIs, while Farkas and É. Kiss (1996) note that they block the comparative reading of superlatives. Additionally, these adverbs appear to also block mono-clausal inverse scope: (39) has no reading in which reporters co-vary with candidates.

(39) Some reporter infuriated very much every candidate who gave a speech at this rally.

An account of these facts will have to be left for further research.

The conclusions we draw from the above discussion are the following. First, inverse scope of distributive universals is limited to (extended) coarguments, rather than to clause-mates. Scope limits are thus seen as sensitive 
accessible function, variables in semantic structure are indexed with the function that fixes their value. The functional index borne by a variable must be accessible to the variable in question. The scope ambiguity of a sentence such as Every nurse examined a patient is due to the fact that the indefinite may be evaluated either by $f$ or by the functions $f^{\prime \prime} \in F^{\prime \prime}$, the former being always accessible, the latter being accessible because the indefinite is in the NS of a quantificational structure whose Restrictor is contributed by a distributive universal. The variable contributed by the indefinite is indexed by $f$ in the former case, and by $f^{\prime \prime}$ in the latter. If $x$ is indexed by $f^{\prime \prime}$, and $f^{\prime \prime}$ extends some functions $f^{\prime}, f^{\prime \prime}$ extends $f^{\prime}$ with respect to $x$.

Now the question of limits on the scope of the universal reduces to the question of when the functions $F^{\prime \prime}$ become available for the interpretation of an indefinite. The accessibility conditions are given in (33):

(33) a. The functions $F^{\prime \prime}$ introduced by a distributive universal binding a variable $x$ are accessible to any variable $y$ introduced by an indefinite in the c-commanding domain of the universal.

b. The functions $F^{\prime \prime}$ introduced by a distributive universal binding a variable $x$ are accessible to a variable $y$ if $x$ and $y$ are co-arguments in some relation $R$.

Condition (33a) accounts for the possibility of direct scope in sentences like $(34 a, b)$; (33b) accounts for the possibility of clause internal inverse scope in cases like (34c).

$$
\begin{array}{ll}
\text { a. } & \text { Every nurse }_{f} \text { examined a patient } \\
\text { b. } & \text { Every nurse }_{f} \text { thought that Dr. Jones cured a patient } \\
\text { c. } & \text { A nurse }_{\left(f^{\prime}\right)} \text { examined every patient } \\
\text {. }
\end{array}
$$

(The subscript $\left(f^{\prime \prime}\right)$ on an indefinite indicates the possibility of the variable introduced by it to be indexed by $f^{\prime \prime}$, and $f^{\prime}$ on the universal indicates that the variable introduced by it must be evaluated by the functions in $F^{\circ}$ ). The wide scope reading of the indefinite with respect to the universal is given by the choice of $f$ as the index of the indefinite, a choice that is assumed to always be available for ordinary indefinites. A universal cannot take an indefinite within its scope when the indefinite is not a co-argument of the universal, or is not in its c-commanding domain because, in that case, neither (33a) nor (33b) are met. The indexing of the indefinite by $f^{\prime \prime}$ in (35) is thus ruled out:

$$
\text { * A nurse } f^{\prime \prime} \text { said that every patient } f_{f} \text { is cured. }
$$

Under these assumptions, extrawide inverse scope is a natural extension of co-argument inverse scope. Condition (33b) above allows an indefinite to be dependent on a universal when the two are relata in some relation $\mathrm{R}$. This condition is fulfilled in mono-clausal cases, where the predicate of the clause 
names the relation in question, and therefore the universal and the indefinite are co-arguments. Crucially now, the condition in (33b) is also fulfilled in the case of extended co-arguments, where the existence of the relation $\mathrm{R}$ relating the universal and the indefinite is entailed by the predicates the two expressions are arguments of. It is because of this entailment that the variable introduced by the indefinite is an argument of a relation that is distributed over the domain of the universal, which renders the distributive functions $F^{\prime \prime}$ introduced by the universal accessible to the indefinite. ${ }^{7}$ The indexing that gives the extrawide scope reading of the universal in (6) is indicated informally in (36a), and more formally in (36b), where $f^{\prime}$ are the functions introduced by the universal, and $f^{\prime \prime}$ are the functions that extend $f$. The truth conditions of (36b) are given in (36c).

(36) a. A student $f^{\prime \prime}$ made sure that every speaker $f$ had a ride.

b. $\quad$ make sure $\left(y_{f}: \operatorname{student}(\mathrm{y}),{ }_{R} \mathrm{x}_{f}\right.$ : speaker $\left.(\mathrm{x})\right]\left[{ }_{N S}\right.$ has $\left.\left.\operatorname{ride}(\mathrm{x})\right]\right)$

c. The expression in (36b) is true iff there is an $f$ with the following property: every $f^{\prime}(f / x)$ such that $f^{\prime}(x) \in \|$ speaker $\|$ has the property that there is an $f^{\prime \prime}\left(f^{\prime} / y\right)$ such that $f^{\prime \prime}(y) \in \|$ student $\|$ such that $f^{\prime \prime}(y)$ made sure that $f^{\prime}(x)$ has a ride.

The effect of indexing $y$ with $f^{\prime \prime}$, which are functions that extend the functions $f$ 'introduced by the universal, is to have the values of $y$ fixed relative to the values of $x$, hence to have $x$ evaluated prior to $y$. This gives the effect of having the Restrictor in (36b) 'raise over' $y$, or, conversely, of having $y$ 'lowered into' the NS in the sense that the semantic contribution of the Restrictor is made available at the level of the embedding predicate. ${ }^{8}$ The functions contributed by $x$ are accessible to $y$ in the absence of c-command only if there is a relation $\mathrm{R}$ that $x$ and $y$ are arguments of. It is in virtue of this relation that the semantic contribution of the universal is accessible to the indefinite, and therefore the universal is interpreted prior to the indefinite. Priority then is determined not only in view of structural configuration but also in view of (entailed) co-argumenthood.

To sum up, under this approach, an indefinite is within the scope of a universal iff the variable introduced by the latter is dependent on the variable introduced by the former. This dependency is represented at semantic structure by a functional index requiring the dependent variable to be evaluated by functions that extend those introduced by the universal. The question of scope limits on the universal becomes a question of accessibility of dependent functional indices. The accessibility relation is sensitive to co-argumenthood/extended co-argumenthood, the latter involving entailments of the predicates taking the relevant variables as arguments: the functions introduced by a strong distributive are accessible to co-arguments and extended co-arguments of the distributive. 
(1967) and Beghelli and Stowell (1995)):

(ii) Each student ran / sang a song.

(iii) ${ }^{*}$ Each student is intelligent.

7. Invoking entailments coming from the lexical semantics of the relevant predicates is not unique to the cases discussed here. It is an influential thesis that, in some cases, such entailments have to be invoked in order to derive grammaticality for negative polarity items (NPIs) in examples such as I am surprised he has any friends, where the well-formedness of the sentence with any is accounted for in terms of an entailment be surprised gives rise to, namely that I expected that he didn't have any friends (cf. Baker (1970), Linebarger (1980), and Giannakidou (in progress)) for arguments and discussion).

8. If one wanted a strict correspondence between scope possibilities and hierarchy at semantic structure one would need to posit a Restrictor Raising rule sensitive to (extended) co-argumenthood which would convert (33b) into (i):

(i) $\left[_{R} \mathrm{x}_{f}\right.$ : speaker(x)] [ ${ }_{N S}$ make sure $\left(\mathrm{y}_{f^{\prime}}\right.$ : student(y), (has ride $\left.\left.(\mathrm{x})\right)\right]$

The analysis is, of course, compatible with a system in which $Q R$ raises universals in a clause-bounded fashion. For an argument in favor of this position, see Kennedy (to appear) and references therein. If one wants the correspondence between scope and universals to hold at LF, QR would have to be made sensitive to the semantic factors discussed here.

9. For an account of the markedness of inverse scope readings based on QR and economy principles, see Reinhart (1995). Under the view developed here indexing that parallels c-command would count as less marked than one which doesn't.

\section{References}

Abush, D. 1994. The scope of indefinites. In Natural Language Semantics, 2,2:83-115.

Bach, E. \& B. Partee. 198(). Anaphora and semantic structure. In Papers from the Parasession on Anaphora, Chicago Linguistic Society, 1-28

Baker, C. L. 1970. Double negatives. In Linguistic Inquiry 1, 169-86

Beghelli, F. 1993. A minimalist approach to quantifier scope. In NELS 23,

Proceedings of the North East Linguistic Society, University of Ottawa, distributed by GLSA, 6.5-80

Beghelli, F \& T. Stowell. 1995. Distributivity and Negation. Ms. To appear in Ways of Scope Taking. ed. by Anna Szabolcsi. Kluwer Academic Publishers, Dordrecht

Ben-Shalom, D. 1993. Object wide scope and semantic trees. In the Proceedings of Semantics and Linguistic Theory (SALT) 3, 19-37

Dowty, D. 1991. Thematic proto-roles and argument selection. In Language 67, 3: 547-619

Farkas, D.F. 1988. On obligatory control.In Linguistics and Philosophy 11,27-58 Farkas, D.F. 1992. On obviation. In Lexical Matters, ed. by I. Sag and A.

Szabolcsi, CSLI, 85-109 
Farkas, D. F. 1995. Evaluation indices and scope. Ms. To appear in Ways of Scope taking, ed. by A. Szabolcsi. Kluwer Academic Publishers, Dordrecht

Farkas, D. F. and E. Kiss. 1996. On the comparative and absolute reading of superlatives. LRC report 95-04. UCSC.

Fodor J. D. \& I. Sag. 1982. Referential and quantificational indefinites. In Linguistics and Philosophy 5, 355-398

Fox, D. and U. Sauerland. 1995. The illusive scope of universal quantifiers. To appear in the Proceedings of NELS 26

Giannakidou, A. (in progress). Negative Polarity and Semantic Licensing Dependencies. Doctoral dissertation. University of Groningen

Giannakidou, A. \& J. Quer. 1995. Two mechanisms for the licensing of negative indefinites. In FLSM VI, Proceedings of the Sixth Annual Meeting of the Formal Linguistics Society of Mid-America. ed. by L. Gabriele, D. Hardinson \& R. Westmoreland, Indiana University, Bloomington, Indiana, distributed by the IULC, 103-114

Heim, I. 1982. The Semantics of Definite and Indefinite NPs. Doctoral dissertation, Umass, Amherst

Hornstein, N. 1995. Logical Form. Basil Blackwell, Oxford.

Kamp, H. 1981. A theory of truth and discourse representation. In Formal Methods in the Study of Language, ed. by Jeroen Groenendijk, Theo Janssen, and Martin Stokhof, 277-322. Mathematical Center, Amsterdam

Kennedy, C. (to appear). Antecedent contained deletion and the syntax of quantification. To appear in Linguistic Inquiry

Linebarger, M. 1980. The Grammar of Negative Polarity. Doctoral diss., MIT

Liu, F. 1991. Scope Dependency in English and Chinese. Doctoral diss., UCLA

McCawley, J. D. 1980. Everything that linguists have always wanted to know about logic, * but were ashamed to ask. Basil Blackwell, Oxford

Reinhart, T. 1995. Interface Strategies. Ms, University of Utrecht

Reinhart, T. \& E. Reuland. 1993. Reflexivity. In Linguistic Inquiry 24, 657-720

Szabolcsi, A. (to appear). Ways of Scope Taking. Kluwer Academic Publishers, Dordrecht

Vendler, Z. 1967. Each and every, any and all. In Linguistics in Philosophy. Ithaca: Cornell University Press, 7()-96 